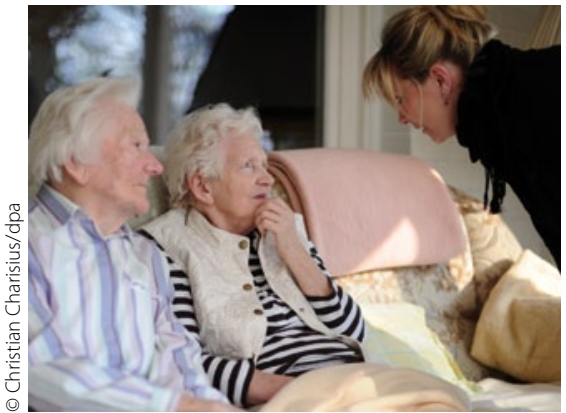

Demenzpatienten haben häufig auch neuropsychiatrische Symptome.

- Ein Großteil der Demenzpatienten leidet unter neuropsychiatrischen Symptomen, so Prof. Horst Herrschaft, Lüneburg. Solche Patienten werden in Demenzstudien aber häufig ausgeschlossen.

Herrschaft und Kollegen untersuchten nun Ginkgo biloba-Extrakt EGb 761 (Tebo$\mathrm{nin}^{\oplus}$ ) bei 410 ambulanten, überwiegend nicht vorbehandelten Patienten mit leichter bis mittelgradiger Demenz (Alzheimer oder vaskulär), die unter neuropsychiatrischen Symptomen litten. Sie waren im Schnitt seit etwa drei Jahren erkrankt. Im Rahmen der doppelblinden und placebokontrollierten Studie wurden sie 24 Wochen lang behandelt. Die Verbesserung

Alzheimer oder vaskuläre Demenz

\title{
Ginkgo-Extrakt bessert Kognition und neuropsychiatrische Symptome
}

der Kognition wurde mit dem SKT-Score (Syndrom-Kurztest), die der Verhaltensauffälligkeiten mit dem NPI-Score (Neuropsychiatric Inventory) ermittelt.

Wie sich zeigte, verbesserten sich die Patienten der Verumgruppe während der Behandlung sowohl in der Kognition (SKT: vom Ausgangswert 15,1 im Schnitt um 2,2 Punkte vs. 0,3 unter Placebo) als auch im Verhalten (NPI: von 16,8 um 4,6 Punkte vs. 2,1 Punkte unter Placebo). Beide Unterschiede waren signifikant $(p<0,001)$. Patienten mit Alzheimer oder vaskulärer Demenz profitierten gleichermaßen.

Auch in vielen sekundären Parametern, mit denen Alltagsfunktionen und Lebensqualität quantifiziert wurden, erzielte die Verum- signifikant bessere Ergebnisse als die Placebogruppe. Wie Prof. Herrschaft betonte, liegt eine der Stärken der Studie darin, dass eine typische, alltagsrelevante Population mit unterschiedlichen Demenzformen untersucht wurde.
Damit bestätigen Herrschaft et al. eine andere Studie der Autorengruppe um Prof. Ralf Ihl, Krefeld. Auch hier war bei 404 Patienten mit leichter bis moderater Demenz unterschiedlicher Genesen und zusätzlichen Verhaltensauffälligkeiten Ginkgo biloba-Extrakt EGb $761^{\circledR}$ über 24 Wochen getestet und für signifikant wirksamer befunden worden (SKT-Differenz: ca. 1,6 Punkte, NPI-Differenz: 3,1 Punkte).

EGb $761^{\circledR}$ beeinflusst mehrere Pathomechanismen, die Demenzen zugrundeliegen, schreibt Herrschaft: Es mindert u. a. die Aggregation und Toxizität von Amyloid Beta und verbessert die Mitochondrienfunktion der Nervenzellen; es setzt die Blutviskosität herab und verbessert die Mikroperfusion.

- Dr. med. Dirk Einecke

Quellen: 1. Herrschaft H., et al. Journal of Psychiatric Research, published online 29.03.2012; 2. Ihl R., Tribanek M., Bachinskaya N., for the GOTADAY Study Group. Pharmacopsychiatry 2012, DOI: 10.1055/s-0031-1291217

\section{Diabetische Nephropathie}

\section{Dosisangepasster DPP-4-Hemmer minimiert Hypoglykämie-Risiko}

— Die Niereninsuffizienz ist eine häufige Spätkomplikation des Diabetes mellitus mit Schrittmacherfunktion für die Krankheitsprogression. Die verringerte Elimination von Medikamenten erfordert zudem besondere Vorsichtmaßnahmen.

Die abnehmende Nierenfunktion bei Diabetes ist ein potenter Risikofaktor für kardiovaskuläre Komplikationen, der die Mortalität erhöht, erinnerte Prof. Dr. Axel Versen, Friedrichshafen. Bei multimorbiden Typ-2-Diabetikern mit Nierenbeteiligung fordert Versen daher ein optimiertes Management der klassischen Risikofaktoren Blutzucker, Blutdruck und Lipide sowie Anstrengungen zur Senkung der Proteinurie. Ausgesprochen wichtig sei dabei auch die Phosphatrestriktion, da die v.a. durch Lebensmittelzusatzstoffe bedingte
Phosphatüberladung weitere endokrine Veränderungen und extraossäre Verkalkungen von Herzklappen und Gefäßen nach sich zieht.

Die eingeschränkte Nierenfunktion birgt zudem die Gefahr der Arzneimittelakkumulation. Dadurch bedingte Nebenwirkungen werden v.a. unter Therapie mit Diuretika, Antibiotika und Antidiabetika beobachtet. Entsprechend steigt bei Nierenschwäche das Hypoglykämierisiko, so Prof. Walter E. Haefeli, Heidelberg.

Die höchste Gefahr von Unterzuckerungen besteht bei Therapie mit Insulinen sowie mit Sulfonylharnstoffen. DPP-4-Hemmer wie Sitagliptin (z. B. Xelevia ${ }^{\circledR}$, Velmetia $\left.{ }^{\oplus}\right)$ schneiden in dieser Hinsicht günstig ab, betonte Haefeli. Dosisangepasst kann Sitagliptin laut Zulassung auch bei Pati- enten mit eingeschränkter Nierenfunktion ohne Akkumulationsgefahr eingesetzt werden: Bei moderater Nierenfunktionsstörung (Kreatinin-Clearance $30-50 \mathrm{ml} /$ $\mathrm{min}$ ) werden statt $100 \mathrm{mg} / \mathrm{d}$ nur $50 \mathrm{mg} / \mathrm{d}$ empfohlen. Bei schwerer bis terminaler Niereninsuffizienz ( $<30 \mathrm{ml} / \mathrm{min}$ ) wird die Dosis nochmals auf $25 \mathrm{mg} / \mathrm{d}$ halbiert.

Ein weiterer Vorteil der DPP-4-Hemmer, der ebenfalls insbesondere bei alten, multimorbiden und nierenkranken Diabetikern zum Tragen kommt, ist laut Haefeli das weitgehende Fehlen klinisch relevanter Arzneimittel-Interaktionen.

- Dr. Katharina Arnheim

Quelle: Symposium „Der multimorbide Typ-2Diabetiker - eine therapeutische Herausforderung", DDG-Kongress, Stuttgart, Mai 2012 (Veranstalter: Berlin-Chemie) 